\title{
GENERATORS OF TRUNCATED SYMMETRIC POLYNOMIALS
}

\author{
FEDERICO GALETTO
}

\begin{abstract}
Adem and Reichstein introduced the ideal of truncated symmetric polynomials to present the permutation invariant subring in the cohomology of a finite product of projective spaces. Building upon their work, I describe a generating set of the ideal of truncated symmetric polynomials in arbitrary positive characteristic, and offer a conjecture for minimal generators.
\end{abstract}

\section{Contents}

1. Introduction

2. Symmetric polynomials and partitions 2

3. Truncated symmetric polynomials 6

4. Generators in positive characteristic $\quad 7$

5. A conjecture for minimal generators 10

6. Acknowledgements 11

References $\quad 11$

\section{INTRODUCTION}

Let $\mathbb{F}$ be a field, and let $R=\mathbb{F}\left[x_{1}, \ldots, x_{n}\right]$ be the polynomial ring in $n$ variables with coefficients in $\mathbb{F}$. The symmetric group $\mathfrak{S}_{n}$ acts on $R$ by permuting the variables. Denote by $R^{\mathfrak{S}_{n}}$ the invariant subring, i.e., the ring of symmetric polynomials. The ideal of truncated symmetric polynomials in $R^{\mathfrak{S}_{n}}$ is defined by

$$
I_{n, d}=\left(x_{1}^{d+1}, \ldots, x_{n}^{d+1}\right) R \cap R^{\mathfrak{S}_{n}} .
$$

The ideal of truncated symmetric polynomials was introduced by A. Adem and Z. Reichstein [1] in the following geometric context. Let $\mathbb{C} P^{d}$ be the complex projective $d$-space, and let $B U(n)$ be the classifying space of the unitary group. The symmetric group $\mathfrak{S}_{n}$ acts on the $n$-fold product $\left(\mathbb{C} P^{d}\right)^{n}$ by permuting the factors. Consider the induced action on the cohomology ring $H^{*}\left(\left(\mathbb{C} P^{d}\right)^{n}, \mathbb{F}\right)$ and let $H^{*}\left(\left(\mathbb{C} P^{d}\right)^{n}, \mathbb{F}\right)^{\mathfrak{S}_{n}}$ be the invariant subring. The two authors show there is a

Date: July 12, 2018.

2010 Mathematics Subject Classification. 13A15, 55R35.

Key words and phrases. symmetric polynomial, positive characteristic, cohomology, classifying space, unitary group. 
map $\left(\mathbb{C} P^{d}\right)^{n} \rightarrow B U(n)$ such that the induced map on cohomology restricts to a ring epimorphism

$$
H^{*}(B U(n), \mathbb{F}) \longrightarrow H^{*}\left(\left(\mathbb{C} P^{d}\right)^{n}, \mathbb{F}\right)^{\mathfrak{S}_{n}}
$$

The cohomology ring of $B U(n)$ can be identified with $R^{\mathfrak{S}_{n}}$, and the kernel of this map can be identified with $I_{n, d}$. Therefore $R^{\mathfrak{S}_{n}} / I_{n, d}$ and $H^{*}\left(\left(\mathbb{C} P^{d}\right)^{n}, \mathbb{F}\right)^{\mathfrak{S}_{n}}$ are isomorphic as ungraded rings (they become isomorphic as graded rings if the grading on $R^{\mathfrak{S}_{n}} / I_{n, d}$ is stretched out by a factor of 2 ). This result was used in the same paper to compute the cohomology of the homotopy fiber for a natural fibration over $B U(n)$.

Adem and Reichstein determined a set of generators of $I_{n, d}$ when the characteristic of $\mathbb{F}$ is zero and when it is strictly bigger than $(n+1) / 2$. Independently, A. Conca, C. Krattenthaler and J. Watanabe [2] identified generators of $I_{n, d}$ working over the complex numbers (although their methods hold over any field of characteristic strictly bigger than $n$ ). In addition, the ideal of truncated symmetric polynomials has generated interest in connection to other topics, such as Lefschetz properties [5] and invariants of Poincaré duality algebras [8].

In this paper, I describe a generating set of $I_{n, d}$ in arbitrary characteristic. Given a partition $\lambda$, let $m_{\lambda} \in R^{\mathfrak{S}_{n}}$ denote the monomial symmetric polynomial associated with $\lambda$. The shorthand notation $\left(\ldots, a^{b}, \ldots\right)$ denotes a partition with the part $a$ repeated $b$ times. The main result reads as follows.

Theorem. Let $t=\max \left\{i \in \mathbb{N} \mid p^{i} \leqslant n\right\}$ and let $q_{0}, \ldots, q_{t} \in \mathbb{N}_{>0}$ be such that, $\forall i \in\{0, \ldots, t\}, n=q_{i} p^{i}+r_{i}$ with $0 \leqslant r_{i}<p^{i}$. For every $i \in\{0, \ldots, t\}$, define an ideal of $R^{\mathfrak{S}_{n}}$

$$
J_{n, d, i}=\left(m_{\left((d+1)^{p^{i}}\right)}, \ldots, m_{\left(\left(d+q_{i}\right)^{p^{i}}\right)}\right) .
$$

Then $I_{n, d}=J_{n, d, 0}+J_{n, d, 1}+\ldots+J_{n, d, t}$.

The next section contains a brief review of symmetric polynomials and some computational results. In section 3, I review the algebraic results of Adem and Reichstein. The proof of the main theorem is in section 4. Finally, in section 5, I present a conjecture for a minimal generating set of the ideal of truncated symmetric polynomials.

\section{Symmetric POLYNOMials AND PARTitions}

Let $\mathbb{F}$ be a field of characteristic $p$. For $n \in \mathbb{N}_{>0}$, set $R=\mathbb{F}\left[x_{1}, \ldots, x_{n}\right]$, the polynomial ring in $n$ indeterminates over $\mathbb{F}$. The symmetric group $\mathfrak{S}_{n}$ acts on $R$ by permuting the variables. Let $R^{\mathfrak{S}_{n}} \subseteq R$ be the invariant subring, i.e., the ring of symmetric polynomials.

Recall that a partition is a sequence

$$
\lambda=\left(\lambda_{1}, \lambda_{2}, \ldots, \lambda_{r}, \ldots\right)
$$


of non-negative integers in non-increasing order:

$$
\lambda_{1} \geqslant \lambda_{2} \geqslant \ldots \geqslant \lambda_{r} \geqslant \ldots
$$

and containing only finitely many nonzero terms. We identify two such sequences which differ only by a string of zeroes at the end. The nonzero numbers $\lambda_{i}$ are called the parts of $\lambda$; the number of parts of $\lambda$ is called the length of $\lambda$ and denoted $l(\lambda)$.

For a partition $\lambda$ with $l(\lambda) \leqslant n$, define the monomial symmetric polynomial on $\lambda$ to be the polynomial,

$$
m_{\lambda}=\sum x_{1}^{\alpha_{1}} \ldots x_{n}^{\alpha_{n}}
$$

summed over all distinct permutations $\alpha=\left(\alpha_{1}, \ldots, \alpha_{n}\right)$ of $\lambda=\left(\lambda_{1}, \ldots, \lambda_{n}\right)$. The polynomials $m_{\lambda}$ are clearly symmetric. Moreover, if $\mathcal{L}_{\leqslant n}$ denotes the set of all partitions with length smaller than or equal to $n$, then $\left\{m_{\lambda} \mid \lambda \in \mathcal{L}_{\leqslant n}\right\}$ is a basis of $R^{\mathfrak{S}_{n}}$ as an $\mathbb{F}$-vector space [6, $\S$ I.2, pp. 18-19]. If $l(\lambda)>n$, then $m_{\lambda}=0$.

Example 2.1. Suppose $n=3$. Then

$$
\begin{aligned}
& m_{(1,1)}=x_{1} x_{2}+x_{1} x_{3}+x_{2} x_{3}, \\
& m_{(2,1)}=x_{1}^{2} x_{2}+x_{1}^{2} x_{3}+x_{1} x_{2}^{2}+x_{1} x_{3}^{2}+x_{2}^{2} x_{3}+x_{2} x_{3}^{2} .
\end{aligned}
$$

Example 2.2 (Power sums).

$$
p_{i}=x_{1}^{i}+\ldots+x_{n}^{i}=m_{(i)}
$$

Example 2.3 (Elementary symmetric polynomials).

$$
e_{i}=\sum_{1 \leqslant j_{1}<\ldots<j_{i} \leqslant n} x_{j_{1}} \ldots x_{j_{i}}=m_{(\underbrace{1, \ldots, 1}_{i \text { times }})}
$$

The following statement contains a multiplication formula for the polynomials $m_{\lambda}$. The proof follows directly from the definitions.

Proposition 2.4. For any $\lambda, \mu \in \mathcal{L}_{\leqslant n}$,

$$
m_{\lambda} m_{\mu}=\sum_{\nu \in \mathcal{L}_{\leqslant n}} c_{\nu} m_{\nu}
$$

where $c_{\nu}$ is the number of different ways to write

$$
\left(\nu_{1}, \ldots, \nu_{n}\right)=\left(\alpha_{1}, \ldots, \alpha_{n}\right)+\left(\beta_{1}, \ldots, \beta_{n}\right)
$$

with $\left(\alpha_{1}, \ldots, \alpha_{n}\right)$ a permutation of $\lambda=\left(\lambda_{1}, \ldots, \lambda_{n}\right)$ and $\left(\beta_{1}, \ldots, \beta_{n}\right)$ a permutation of $\mu=\left(\mu_{1}, \ldots, \mu_{n}\right)$.

Example 2.5. Suppose $n=3$. Then

$$
m_{(1,1)} m_{(2,1)}=m_{(3,2)}+2 m_{(3,1,1)}+2 m_{(2,2,1)},
$$


since

$$
\begin{aligned}
& (3,2,0)=(1,1,0)+(2,1,0), \\
& (3,1,1)=(1,1,0)+(2,0,1)=(1,0,1)+(2,1,0), \\
& (2,2,1)=(0,1,1)+(2,1,0)=(1,0,1)+(1,2,0) .
\end{aligned}
$$

Let $\lambda_{1}, \ldots, \lambda_{k}$ be integers such that

$$
\lambda_{1}>\lambda_{2}>\ldots>\lambda_{k}>0
$$

and let $w_{1}, \ldots, w_{k}$ be positive integers. Denote by $\left(\lambda_{1}^{w_{1}}, \ldots, \lambda_{k}^{w_{k}}\right)$ the partition $\lambda$ having $w_{i}$ parts equal to $\lambda_{i}$ for every $i \in\{1, \ldots, k\}$. The number $w_{i}$ will be referred to as the multiplicity of $\lambda_{i}$ in $\lambda$. In particular, $\lambda_{1}$ will be called the leading part of $\lambda$, denoted by $\operatorname{lp}(\lambda)$, and $w_{1}$ will be called the leading multiplicity of $\lambda$, denoted by $\operatorname{lm}(\lambda)$. Notice $l(\lambda)=w_{1}+\ldots+w_{k}$.

We provide here a list of formulas that will be employed later on. The first one is a classical result by Newton (see [7]).

Proposition 2.6 (Newton's identities). For any $s>n$,

$$
m_{(s)}=\sum_{j=1}^{n}(-1)^{j-1} m_{\left(1^{j}\right)} m_{(s-j)} .
$$

Example 2.7. Let $n=3$ and $s=4$. Then

$$
m_{(4)}=m_{(1)} m_{(3)}-m_{(1,1)} m_{(2)}+m_{(1,1,1)} m_{(1)} .
$$

Lemma 2.8. Let $\lambda=\left(\lambda_{1}^{w_{1}}, \ldots, \lambda_{k}^{w_{k}}\right) \in \mathcal{L}_{\leqslant n}$, with $k>1$. Then

$$
m_{\left(\lambda_{1}^{w_{1}}\right)} m_{\left(\lambda_{2}^{w_{2}}, \ldots, \lambda_{k}^{w_{k}}\right)}=m_{\lambda}+\sum_{\substack{\operatorname{lp}(\mu)>\lambda_{1} \\ \operatorname{lm}(\mu)=w_{1} \\ l(\mu)<l(\lambda)}} a_{\mu} m_{\mu}+\sum_{\substack{\operatorname{lp}(\nu)>\lambda_{1} \\ \operatorname{lm}(\nu)<w_{1}}} b_{\nu} m_{\nu},
$$

for some $a_{\mu}, b_{\nu} \in \mathbb{F}$.

Proof. Expanding the product on the left hand side in the basis of monomial symmetric polynomials, we obtain

$$
m_{\left(\lambda_{1}^{w_{1}}\right)} m_{\left(\lambda_{2}^{w_{2}}, \ldots, \lambda_{k}^{w_{k}}\right)}=\sum_{\theta \in \mathcal{L}_{\leqslant n}} c_{\theta} m_{\theta}
$$

for some $c_{\theta} \in \mathbb{F}$. We proceed to identify the partitions $\theta$ for which the coefficient $c_{\theta}$ may be non-zero.

Consider first the case when $\theta=\lambda$. The only way to obtain the partition $\lambda$ as a sum of permutations of $\left(\lambda_{1}^{w_{1}}\right)$ and $\left(\lambda_{2}^{w_{2}}, \ldots, \lambda_{k}^{w_{k}}\right)$ is

$$
\left(\lambda^{w_{1}}, 0, \ldots, 0\right)+\left(0^{w_{1}}, \lambda_{2}^{w_{2}}, \ldots, \lambda_{k}^{w_{k}}, 0, \ldots, 0\right),
$$

where, abusing notation, $0^{w_{1}}$ means the entry 0 is repeated $w_{1}$ times. Thus $c_{\theta}=1$ by Proposition 2.4. 
Now suppose $\theta \neq \lambda$ and $c_{\theta} \neq 0$. Since $\lambda_{1}>\ldots>\lambda_{k}>0, \operatorname{lp}(\theta)=\lambda_{1}+\lambda_{j}$ for some $j \in\{2, \ldots, k\}$. Note that $\lambda_{1}+\lambda_{j}>\lambda_{1}$, hence $\operatorname{lp}(\theta)>\lambda_{1}$.

If there is an index $j \in\{2, \ldots, k\}$ such that $w_{j} \geqslant w_{1}$, then we have a partition

$$
\begin{aligned}
\mu & =\left(\left(\lambda_{1}+\lambda_{j}\right)^{w_{1}}, \lambda_{2}^{w_{2}}, \ldots, \lambda_{j}^{w_{j}-w_{1}}, \ldots, \lambda_{k}^{w_{k}}\right)= \\
& =\left(\lambda_{1}^{w_{1}}, 0, \ldots, 0\right)+\left(\lambda_{j}^{w_{1}}, \lambda_{2}^{w_{2}}, \ldots, \lambda_{j}^{w_{j}-w_{1}}, \ldots, \lambda_{k}^{w_{k}}\right)
\end{aligned}
$$

Therefore, by Proposition 2.4, the element $m_{\mu}$ may appear with non-zero coefficient in our expansion. Observe that $\operatorname{lm}(\mu)=w_{1}$ and $\mu$ has length

$$
w_{1}+w_{2}+\ldots+\left(w_{j}-w_{1}\right)+\ldots+w_{k}=\sum_{j=2}^{k} w_{j}<l(\lambda) .
$$

Similarly, we may obtain other partitions $\mu$ with the same properties. We collect them all in a single summation as in the statement of the lemma.

The remaining terms are of the form $b_{\nu} m_{\nu}$ where, as observed, $\operatorname{lp}(\nu)>\lambda_{1}$ and, in addition, $\operatorname{lm}(\nu)<w_{1}$. We collect them in a single summation to obtain the desired formula.

Example 2.9. Let $n=6$ and $\lambda=(3,3,2,2,1)$. Then

$$
\begin{aligned}
m_{(3,3)} m_{(2,2,2,1)}= & m_{(3,3,2,2,2,1)}+ \\
& +m_{(5,5,2,1)}+ \\
& +m_{(5,4,2,2)}+m_{(5,3,2,2,1)}+m_{(4,3,2,2,2)} .
\end{aligned}
$$

Lemma 2.10. Let $\lambda=\left(\lambda_{1}^{w_{1}}, \ldots, \lambda_{k}^{w_{k}}\right) \in \mathcal{L}_{\leqslant n}$. If $s$ is a positive integer such that $s+l(\lambda) \leqslant n$, then

$$
m_{\left(\lambda_{1}^{s}\right)} m_{\lambda}=\left(\begin{array}{c}
s+w_{1} \\
s
\end{array}\right) m_{\left(\lambda_{1}^{s+w_{1}}, \lambda_{2}^{w_{2}}, \ldots, \lambda_{k}^{w_{k}}\right)}+\sum_{\substack{\operatorname{lp}(\mu)>\lambda_{1} \\
\ln (\mu)=s \\
l(\mu)=l(\lambda)}} a_{\mu} m_{\mu}+\sum_{\substack{\operatorname{lp}(\nu)>\lambda_{1} \\
\operatorname{lm}(\nu)<s}} b_{\nu} m_{\nu}
$$

for some $a_{\mu}, b_{\nu} \in \mathbb{F}$.

Proof. The proof follows from the same reasoning used to prove Lemma 2.8. The only aspect that requires further clarification is the binomial coefficient that appears in front of

$$
m_{\left(\lambda_{1}^{s+w_{1}}, \lambda_{2}^{w_{2}}, \ldots, \lambda_{k}^{w_{k}}\right)}
$$

This polynomial arises whenever a permutation of $\left(\lambda_{1}^{s}, 0, \ldots, 0\right)$ is chosen that has $s$ of its first $s+w_{1}$ entries occupied by $\lambda_{1}$, and the remaining ones by 0 , while simultaneously a permutation of $\left(\lambda_{1}^{w_{1}}, \ldots, \lambda_{k}^{w_{k}}\right)$ is chosen that has the same $s$ among its first $s+w_{1}$ entries occupied by 0 , and the remaining ones by $\lambda_{1}$. The number of times this occurs is the number of ways to choose $s$ entries among the first $s+w_{1}$. 
Example 2.11. Let $n=6, \lambda=\left(3^{2}, 2,1\right)$ and $s=2$. Then

$$
\begin{aligned}
m_{\left(3^{2}\right)} m_{\left(3^{2}, 2,1\right)}= & 6 m_{\left(3^{4}, 2,1\right)}+ \\
& +m_{\left(6^{2}, 2,1\right)}+ \\
& +\left(m_{(6,5,3,1)}+m_{(6,4,3,2)}+2 m_{\left(6,3^{2}, 2,1\right)}+\right. \\
& \left.+m_{\left(5,4,3^{2}\right)}+3 m_{\left(5,3^{3}, 1\right)}+3 m_{\left(4,3^{3}, 2\right)}\right) .
\end{aligned}
$$

We are interested in the case when the binomial coefficient appearing in Lemma 2.10 is invertible in $\mathbb{F}$. This is always the case when $\mathbb{F}$ has characteristic zero. The following result is helpful when $\mathbb{F}$ has characteristic $p>0$.

Theorem 2.12 (Lucas). Let $u$ and $v$ be non-negative integers, $p$ a prime, and

$$
\begin{aligned}
& u=u_{t} p^{t}+u_{t-1} p^{t-1}+\ldots+u_{1} p+u_{0}, \\
& v=v_{t} p^{t}+v_{t-1} p^{t-1}+\ldots+v_{1} p+v_{0},
\end{aligned}
$$

the base $p$ expansions of $u$ and $v$. Then

$$
\left(\begin{array}{l}
u \\
v
\end{array}\right) \equiv \prod_{j=0}^{t}\left(\begin{array}{l}
u_{j} \\
v_{j}
\end{array}\right) \quad(\bmod p) .
$$

A proof can be found in $[3, \S 6]$.

\section{TRunCATED SYMmetric POLYNOMIALS}

For a given non-negative integer $d$, consider the ideal $\left(x_{1}^{d+1}, \ldots, x_{n}^{d+1}\right) \subseteq R$. Denote by $I_{n, d}$ the intersection

$$
\left(x_{1}^{d+1}, \ldots, x_{n}^{d+1}\right) \cap R^{\mathfrak{S}_{n}} .
$$

Since $R^{\mathfrak{S}_{n}}$ is a subring of $R, I_{n, d}$ is an ideal of $R^{\mathfrak{S}_{n}}$. Call $I_{n, d}$ the ideal of truncated symmetric polynomials.

In this section, I will recall ideas and results previously presented in [1]. For the convenience of the reader, all statements and proofs will be phrased in the language and notation of this paper.

The following proposition was recorded as a simple observation without proof in the paper of Adem and Reichstein. I fill in the details of the proof below.

\section{Proposition 3.1.}

(a) Let $\lambda \in \mathcal{L}_{\leqslant n}$. Then $m_{\lambda} \in I_{n, d}$ if and only if $\operatorname{lp}(\lambda) \geqslant d+1$.

(b) The set

$$
\left\{m_{\lambda} \mid \lambda \in \mathcal{L}_{\leqslant n}, \operatorname{lp}(\lambda) \geqslant d+1\right\}
$$

spans $I_{n, d}$ over $\mathbb{F}$. 
Proof. (a) Let $\lambda=\left(\lambda_{1}, \ldots, \lambda_{n}\right)$. If $\operatorname{lp}(\lambda) \geqslant d+1$, then $\lambda_{1} \geqslant d+1$. Hence, for any permutation $\left(\alpha_{1}, \ldots, \alpha_{n}\right)$ of $\left(\lambda_{1}, \ldots, \lambda_{n}\right)$, there is some index $i$ such that $\alpha_{i} \geqslant d+1$ and $x_{1}^{\alpha_{1}} x_{2}^{\alpha_{2}} \ldots x_{n}^{\alpha_{n}} \in\left(x_{1}^{d+1}, \ldots, x_{n}^{d+1}\right)$. Therefore $m_{\lambda} \in I_{n, d}$.

Vice versa, assume $m_{\lambda} \in I_{n, d}$. Then $m_{\lambda} \in\left(x_{1}^{d+1}, \ldots, x_{n}^{d+1}\right)$. Note that $\left\{x_{1}^{d+1}, \ldots, x_{n}^{d+1}\right\}$ is the reduced Gröbner basis of the ideal $\left(x_{1}^{d+1}, \ldots, x_{n}^{d+1}\right)$ in the lexicographic term ordering on $R$. Therefore the leading term of $m_{\lambda}$ is divisible by $x_{i}^{d+1}$ for some $i$. Since the leading term of $m_{\lambda}$ is $x_{1}^{\lambda_{1}} x_{2}^{\lambda_{2}} \ldots x_{n}^{\lambda_{n}}$, it follows that $\operatorname{lp}(\lambda)=\lambda_{1} \geqslant \lambda_{i} \geqslant d+1$.

(b) Let $0 \neq g \in I_{n, d}$. Since the monomial symmetric polynomials form a basis of $R^{\mathfrak{S}_{n}}$ over $\mathbb{F}, g$ can be written uniquely as a linear combination

$$
g=\sum_{\theta \in \mathcal{L}_{\leqslant n}} c_{\theta} m_{\theta}
$$

for some $c_{\theta} \in \mathbb{F}$. The set $\left\{\theta \in \mathcal{L}_{\leqslant n} \mid c_{\theta} \neq 0\right\}$ is nonempty, finite, and totally ordered lexicographically. Let $\lambda$ be its maximum. Then $x_{1}^{\lambda_{1}} x_{2}^{\lambda_{2}} \ldots x_{n}^{\lambda_{n}}$ is the leading term of $g$. Reasoning as in part (a), deduce that $\operatorname{lp}(\lambda) \geqslant d+1$. Now repeat this process with $g-c_{\lambda} m_{\lambda}$ to show that the remaining $m_{\theta}$ in the expression of $g$ also have leading part at least $d+1$. Therefore $g$ is in the span of the desired set.

The next result corresponds to [1, Lemma 5.2].

Proposition 3.2. Let $J_{n, d}=\left(m_{(d+1)}, \ldots, m_{(d+n)}\right) \subseteq R^{\mathfrak{S}_{n}}$. Then $J_{n, d}$ contains every $m_{\lambda}$ with $\operatorname{lp}(\lambda) \geqslant d+1$ and $\operatorname{lm}(\lambda) \in \mathbb{F}^{\times}$.

The last result of this section is an immediate consequence of Proposition 3.2. It is part of Adem and Reichstein's main theorem [1, Thm. 5.1(a)].

Corollary 3.3. If $p=0$ or $n<p$, then $I_{n, d}=\left(m_{(d+1)}, \ldots, m_{(d+n)}\right)$.

\section{Generators in positive characteristic}

As Adem and Reichstein already noticed, the polynomials $m_{(d+1)}, \ldots, m_{(d+n)}$ alone do not generate $I_{n, d}$ when $n \geqslant p$. For $n \leqslant 2 p-1$, the two authors showed that taking $m_{\left((d+1)^{p}\right)}$ in addition to $m_{(d+1)}, \ldots, m_{(d+n)}$ is enough to generate $I_{n, d}$ [1, Thm. 5.1(b)]. The goal of this section is to describe a set of generators of $I_{n, d}$ in arbitrary positive characteristic. Throughout this section, $p>0$ will be assumed.

The following is a generalization of Newton's identities.

Lemma 4.1. Consider $h \leqslant n$ and let $q \in \mathbb{N}_{>0}$ be such that $n=q h+r$ with $0 \leqslant r<h$. For every $s \in \mathbb{N}_{>0}$,

$$
\sum_{j=1}^{q}(-1)^{j-1} m_{\left(1^{j h}\right)} m_{\left((s+q+1-j)^{h}\right)}=m_{\left((s+q+1)^{h}\right)}+\sum_{\substack{\operatorname{lp}(\mu)>s+1 \\ \operatorname{lm}(\mu)<h}} a_{\mu} m_{\mu},
$$


for some $a_{\mu} \in \mathbb{F}$.

Proof. When $j \in\{1, \ldots, q-1\}$, the product $m_{\left(1^{j h}\right)} m_{\left((s+q+1-j)^{h}\right)}$ expands to

$$
m_{\left((s+q+2-j)^{h}, 1^{(j-1) h}\right)}+m_{\left((s+q+1-j)^{h}, 1^{j h}\right)}+\sum_{\substack{\operatorname{lp}(\mu)=s+q+2-j \\ \operatorname{lm}(\mu)<h}} a_{\mu, j} m_{\mu},
$$

for some $a_{\mu, j} \in \mathbb{F}$. When $j=q$, the same product becomes $m_{\left(1^{q h}\right)} m_{\left((s+1)^{h}\right)}$ which expands to

$$
m_{\left((s+2)^{h}, 1^{(q-1) h}\right)}+\sum_{\substack{\operatorname{lp}(\mu)=s+2 \\ \operatorname{lm}(\mu)<h}} a_{\mu, q} m_{\mu}
$$

for some $a_{\mu, q} \in \mathbb{F}$. Summing over $j$ with alternating signs, all monomial symmetric polynomials with leading multiplicity $h$ cancel out except for $m_{\left((s+q+1)^{h}\right)}$ which occurs when $j=1$. The remaining terms can be combined in a single summation to give the formula above.

Proposition 4.2. Let $i \in \mathbb{N}$ be such that $p^{i} \leqslant n$ and let $q \in \mathbb{N}_{>0}$ be such that $n=q p^{i}+r$ with $0 \leqslant r<p^{i}$. Let $K$ be an ideal of $R^{\mathfrak{S}_{n}}$ such that

$$
K \supseteq\left\{m_{\lambda} \mid \lambda \in \mathcal{L}_{\leqslant n}, \operatorname{lp}(\lambda) \geqslant d+1, \operatorname{lm}(\lambda)<p^{i}\right\} .
$$

If $J=\left(m_{\left((d+1)^{p^{i}}\right)}, \ldots, m_{\left((d+q)^{p^{i}}\right)}\right)$, then $J+K$ contains every $m_{\lambda}$ with $\operatorname{lp}(\lambda) \geqslant d+1$ and $\operatorname{lm}(\lambda) \leqslant p^{i}$.

Proof. If $\lambda \in \mathcal{L}_{\leqslant n}$ has $\operatorname{lp}(\lambda) \geqslant d+1$ and $\operatorname{lm}(\lambda)<p^{i}$, then, by the hypothesis, $m_{\lambda} \in K \subseteq J+K$.

Suppose $\operatorname{lp}(\lambda) \geqslant d+1$ and $\operatorname{lm}(\lambda)=p^{i}$. The proof that $m_{\lambda} \in J+K$ is by induction on $l(\lambda)$. For the base case, assume $l(\lambda)=p^{i}$, i.e., $m_{\lambda}=m_{\left(\lambda_{1}^{p^{i}}\right)}$ for some $\lambda_{1} \geqslant d+1$. By definition, $m_{\left(\lambda_{1}^{p^{i}}\right)} \in J \subseteq J+K$, when $d+1 \leqslant \lambda_{1} \leqslant d+q$. If $\lambda_{1}>d+q$, then we may write $\lambda_{1}=s+q+1$, for some $s \geqslant d$. Applying Lemma 4.1 with $h=p^{i}$,

$$
\sum_{j=1}^{q}(-1)^{j-1} m_{\left(1^{j p^{i}}\right)} m_{\left((s+q+1-j)^{p^{i}}\right)}=m_{\left((s+q+1)^{p^{i}}\right)}+\sum_{\substack{\operatorname{lp}(\mu)>s+1 \\ \operatorname{lm}(\mu)<p^{i}}} a_{\mu} m_{\mu},
$$

for some $a_{\mu} \in \mathbb{F}$. The summation on the right is in $K \subseteq J+K$ by the hypothesis. Proceeding by induction on $s$, the left hand side belongs to $J+K$. Hence $m_{\left((s+q+1)^{p^{i}}\right)} \in J+K$.

For the inductive step, assume $\lambda=\left(\lambda_{1}^{p^{i}}, \lambda_{2}^{w_{2}}, \ldots, \lambda_{k}^{w_{k}}\right)$ with $l(\lambda)>p^{i}$ and $\lambda_{1} \geqslant$ $d+1$. By Lemma 2.8,

$$
m_{\left(\lambda_{1}^{p^{i}}\right)} m_{\left(\lambda_{2}^{w_{2}}, \ldots, \lambda_{k}^{w_{k}}\right)}=m_{\lambda}+\sum_{\substack{\operatorname{lp}(\mu)>\lambda_{1} \\ \operatorname{lm}(\mu)=p^{i} \\ l(\mu)<l(\lambda)}} a_{\mu} m_{\mu}+\sum_{\substack{\operatorname{lp}(\nu)>\lambda_{1} \\ \operatorname{lm}(\nu)<p^{i}}} b_{\nu} m_{\nu}
$$


for some $a_{\mu}, b_{\nu} \in \mathbb{F}$. By the base case, $m_{\left(\lambda_{1}^{p^{i}}\right)} \in J+K$. On the right hand side, the first summation belongs to $J+K$ by the inductive hypothesis and the second one belongs to $K \subseteq J+K$ by the hypothesis. Thus $m_{\lambda} \in J+K$.

We are now ready to exhibit a set of generators for the ideal $I_{n, d}$.

Theorem 4.3. Let $t=\max \left\{i \in \mathbb{N} \mid p^{i} \leqslant n\right\}$ and let $q_{0}, \ldots, q_{t} \in \mathbb{N}_{>0}$ be such that, $\forall i \in\{0, \ldots, t\}, n=q_{i} p^{i}+r_{i}$ with $0 \leqslant r_{i}<p^{i}$. For every $i \in\{0, \ldots, t\}$, define an ideal of $R^{\mathfrak{S}_{n}}$

$$
J_{n, d, i}=\left(m_{\left((d+1)^{p^{i}}\right)}, \ldots, m_{\left(\left(d+q_{i}\right)^{p^{i}}\right)}\right) .
$$

Then $I_{n, d}=J_{n, d, 0}+J_{n, d, 1}+\ldots+J_{n, d, t}$.

Example 4.4. Let $p=2, n=7$, and $d=1$. Then

$$
\begin{aligned}
& J_{7,1,0}=\left(m_{(2)}, m_{(3)}, m_{(4)}, m_{(5)}, m_{(6)}, m_{(7)}, m_{(8)}\right), \\
& J_{7,1,1}=\left(m_{\left(2^{2}\right)}, m_{\left(3^{2}\right)}, m_{\left(4^{2}\right)}\right), \\
& J_{7,1,2}=\left(m_{\left(2^{4}\right)}\right),
\end{aligned}
$$

and $I_{7,1}=J_{7,1,0}+J_{7,1,1}+J_{7,1,2}$.

Proof. Define auxiliary ideals by setting

$$
K_{i}:=J_{n, d, 0}+J_{n, d, 1}+\ldots+J_{n, d, i}
$$

for $i \in\{0, \ldots, t\}$. With this notation, the thesis becomes $I_{n, d}=K_{t}$. It is clear that $K_{0} \subseteq K_{1} \subseteq \ldots \subseteq K_{t}$. Moreover, $K_{t} \subseteq I_{n, d}$ by Proposition 3.1(a). In light of Proposition 3.1(b), the inclusion $I_{n, d} \subseteq K_{t}$ will follow if one can show that $K_{t}$ contains every polynomial $m_{\lambda}$ with $\operatorname{lp}(\lambda) \geqslant d+1$.

Claim: $\forall i \in\{0, \ldots, t\}$, the inclusion

$$
K_{i} \supseteq\left\{m_{\lambda} \mid \lambda \in \mathcal{L}_{\leqslant n}, \operatorname{lp}(\lambda) \geqslant d+1, \operatorname{lm}(\lambda)<p^{i+1}\right\}
$$

holds.

The claim will be proven by induction on $i$. For $i=0$, note that

$$
K_{0}=J_{n, d, 0}=\left(m_{(d+1)}, \ldots, m_{(d+n)}\right) .
$$

Hence the claim is an immediate consequence of Proposition 3.2.

Assume $i>0$. By the inductive hypothesis,

$$
K_{i-1} \supseteq\left\{m_{\lambda} \mid \lambda \in \mathcal{L}_{\leqslant n}, \operatorname{lp}(\lambda) \geqslant d+1, \operatorname{lm}(\lambda)<p^{i}\right\} .
$$

By definition, $K_{i}=J_{n, d, i}+K_{i-1}$. Hence, by Proposition 4.2 ,

$$
K_{i} \supseteq\left\{m_{\lambda} \mid \lambda \in \mathcal{L}_{\leqslant n}, \operatorname{lp}(\lambda) \geqslant d+1, \operatorname{lm}(\lambda) \leqslant p^{i}\right\} .
$$

It remains to show $K_{i}$ contains all monomial symmetric polynomials $m_{\lambda}$ with $p^{i}<\operatorname{lm}(\lambda)<p^{i+1}$. Let $\lambda=\left(\lambda_{1}^{w_{1}}, \ldots, \lambda_{k}^{w_{k}}\right) \in \mathcal{L}_{\leqslant n}$ with $\lambda_{1} \geqslant d+1$ and $p^{i}<w_{1}<$ $p^{i+1}$. The base $p$ expansion of $w_{1}$ is

$$
w_{1}=h_{i} p^{i}+h_{i-1} p^{i-1}+\ldots+h_{1} p+h_{0}
$$


where $0 \leqslant h_{j}<p, \forall j \in\{0, \ldots, i\}$, and $h_{i} \neq 0$. Set $z=h_{i-1} p^{i-1}+\ldots+h_{0}$, so that $w_{1}=h_{i} p^{i}+z$. By Lemma 2.10,

$$
m_{\left(\lambda_{1}^{p^{i}}\right)} m_{\left(\lambda_{1}^{\left(h_{i}-1\right) p^{i}+z}, \lambda_{2}^{w_{2}}, \ldots, \lambda_{k}^{w_{k}}\right)}=\left(\begin{array}{c}
w_{1} \\
p^{i}
\end{array}\right) m_{\lambda}+\sum_{\substack{\operatorname{lp}(\mu)>\lambda_{1} \\
\operatorname{lm}(\mu)=p^{i} \\
l(\mu)=l(\lambda)}} a_{\mu} m_{\mu}+\sum_{\substack{\operatorname{lp}(\nu)>\lambda_{1} \\
\operatorname{lm}(\nu)<p^{i}}} b_{\nu} m_{\nu},
$$

for some $a_{\mu}, b_{\nu} \in \mathbb{F}$. By (1), $m_{\left(\lambda_{1}^{p^{i}}\right)}$ and the two summations on the right hand side all belong to $K_{i}$. Moreover, by Lucas' theorem,

$$
\left(\begin{array}{c}
w_{1} \\
p^{i}
\end{array}\right) \equiv\left(\begin{array}{c}
h_{i} \\
1
\end{array}\right) \prod_{j=0}^{i-1}\left(\begin{array}{c}
h_{j} \\
0
\end{array}\right) \equiv h_{i} \quad(\bmod p) .
$$

Since $0<h_{i}<p,\left(\begin{array}{c}w_{1} \\ p^{i}\end{array}\right) \in \mathbb{F}^{\times}$; therefore $m_{\lambda} \in K_{i}$. This concludes the proof of the claim.

When $i=t$, the claim gives

$$
K_{t} \supseteq\left\{m_{\lambda} \mid \lambda \in \mathcal{L}_{\leqslant n}, \operatorname{lp}(\lambda) \geqslant d+1, \operatorname{lm}(\lambda)<p^{t+1}\right\} .
$$

If $\lambda \in \mathcal{L}_{\leqslant n}$ and $\operatorname{lp}(\lambda) \geqslant d+1$, the inequality $\operatorname{lm}(\lambda) \leqslant l(\lambda) \leqslant n<p^{t+1}$ implies $m_{\lambda} \in K_{t}$. Therefore $I_{n, d}=K_{t}$.

\section{A CONJECTURE For Minimal Generators}

In this section, I consider the problem of describing minimal generators of the ideal $I_{n, d}$. When $p=0$ or $p>n$ !, a solution was already given by Adem and Reichstein (see [1, Thm. 6.1(a)] and [1, Lemma 6.2(c)]); namely, the generators of Corollary 3.3 are minimal.

Theorem 4.3 describes a set of generators of $I_{n, d}$ for arbitrary $p>0$. In general, this set is not minimal.

Example 5.1. Let $p=2, n=7$ and $d=1$. Refer to Example 4.4 for a generating set of $I_{7,1}$. Notice that

$$
\left(m_{(2)}\right)^{2}=\left(\sum_{i=1}^{7} x_{i}^{2}\right)^{2}=\sum_{i=1}^{7} x_{i}^{4}=m_{(4)},
$$

making $m_{(4)}$ redundant as a generator. Similarly, $m_{(6)}$ and $m_{(8)}$ can be discarded because $m_{(6)}=\left(m_{(3)}\right)^{2}$ and $m_{(8)}=\left(m_{(4)}\right)^{2}$.

A similar computation yields

$$
\left(m_{\left(2^{2}\right)}\right)^{2}=\left(\sum_{1 \leqslant i<j \leqslant 7} x_{i}^{2} x_{j}^{2}\right)^{2}=\sum_{1 \leqslant i<j \leqslant 7} x_{i}^{4} x_{j}^{4}=m_{\left(4^{2}\right)} .
$$

Therefore $m_{\left(4^{2}\right)}$ is also redundant. 
In general, when $p>0$, we have

$$
\left(m_{\left(s^{k}\right)}\right)^{p}=m_{\left((p s)^{k}\right)} .
$$

This observation allows to trim the generating set of Theorem 4.3, leading to the following.

Conjecture. Let $t=\max \left\{i \in \mathbb{N} \mid p^{i} \leqslant n\right\}$ and let $q_{0}, \ldots, q_{t} \in \mathbb{N}_{>0}$ be such that, $\forall i \in\{0, \ldots, t\}, n=q_{i} p^{i}+r_{i}$ with $0 \leqslant r_{i}<p^{i}$. For every $i \in\{0, \ldots, t\}$, define the set

$$
\mathcal{J}_{n, d, i}=\left\{m_{\left((d+h)^{p^{i}}\right)} \mid h \in\left\{1, \ldots, q_{i}\right\} \text { and } \forall j \in\{1, \ldots, h\} d+h \neq p(d+j)\right\} .
$$

Then $\mathcal{J}_{n, d, 0} \cup \mathcal{J}_{n, d, 1} \cup \ldots \cup \mathcal{J}_{n, d, t}$ is a minimal set of generators of $I_{n, d}$.

Essentially, this conjecture claims that the only possible relations among the generators of Theorem 4.3 are of the kind given in equation (2). For small values of $p, n$ and $d$, the conjecture has been verified using the software Macaulay2 [4].

\section{AcKnowledgements}

I am indebted to the anonymous referee, whose useful comments and suggestions helped improve the quality of this manuscript. While working on this project, I was partially supported by an NSERC grant.

\section{REFERENCES}

[1] A. Adem and Z. Reichstein. Bundles, cohomology and truncated symmetric polynomials. Doc. Math., 15:1029-1047, 2010.

[2] A. Conca, C. Krattenthaler, and J. Watanabe. Regular sequences of symmetric polynomials. Rend. Semin. Mat. Univ. Padova, 121:179-199, 2009.

[3] A. Granville. Arithmetic properties of binomial coefficients. I. Binomial coefficients modulo prime powers. In Organic mathematics (Burnaby, BC, 1995), volume 20 of CMS Conf. Proc., pages 253-276. Amer. Math. Soc., Providence, RI, 1997.

[4] D. R. Grayson and M. E. Stillman. Macaulay2, a software system for research in algebraic geometry. Available at http://www.math.uiuc.edu/Macaulay2/.

[5] T. Harima and J. Watanabe. The strong Lefschetz property for Artinian algebras with nonstandard grading. J. Algebra, 311(2):511-537, 2007.

[6] I. G. Macdonald. Symmetric functions and Hall polynomials. Oxford Mathematical Monographs. The Clarendon Press, Oxford University Press, New York, second edition, 1995. With contributions by A. Zelevinsky, Oxford Science Publications.

[7] D. G. Mead. Newton's identities. Amer. Math. Monthly, 99(8):749-751, 1992.

[8] L. Smith. Group actions on Poincaré duality algebras and Lefschetz properties. J. Pure Appl. Algebra, 219(8):3535-3542, 2015.

Federico Galetto, Mcmaster University, 1280 Main St W, 407 Hamilton Hall, HamilTON, ON, L8S 4K1, CANADA

E-mail address: galettof@math.mcmaster.ca

$U R L:$ http://math.galetto.org 